\title{
Development of a Work Style Compass and Predicting Research Productivity of Agricultural Scientists
}

\author{
Sudipta Paul ${ }^{1 *}$, K. Vijayaragavan ${ }^{2}$, Premlata Singh ${ }^{3}$ and Rajarshi Roy Burman ${ }^{4}$ \\ ${ }^{1-4}$ Division of Agricultural Extension, Indian Agricultural Research Institute, Pusa Campus, New Delhi (110 012), India \\ "Presently Agricultural Extension, Zonal Project Directorate, Zone-III, Umiam (Barapani), Meghalaya (793 103), India
}

\section{Article History}

Manuscript No. AR843

Received in $11^{\text {th }}$ July, 2014

Received in revised form $16^{\text {th }}$ December, 2014

Accepted in final form $27^{\text {th }}$ January, 2015

\section{Correspondence to}

${ }^{*}$ E-mail: sudiptaiari@gmail.com

\section{Keywords}

Work style, research productivity, agricultural scientist

\begin{abstract}
In light of increasing thrust of the public agricultural research system of India to enhance research productivity of its scientists, gauging scientists' productivity and its personality correlates has been felt crucial. In this context, devising a simpler method to predict research productivity based upon individual work styles had been aimed in the present study. Randomly drawn two hundred agricultural scientists from different cadres of two differently performing agricultural institutes of the country constituted the sample of the study. A work style compass was designed by taking a measurement of individual work styles of the pooled sample after developing and pretesting Likert type scales. A research productivity index was developed to obtain productivity scores of scientists. A correlation and stepwise multiple regression analysis helped in deriving a suitable model to predict research productivity through the identified work style variables. The findings of the study confirmed a strong and positive relationship between research productivity and individual work styles. The results indicate to the crucial need for organizing work style sensitization workshops for the scientists and undertaking organization development interventions at individual institute level at regular intervals.
\end{abstract}

\section{Introduction}

Agricultural research across the world has been gaining more and more importance, day by day, due to the potential threats posed by the challenges like stagnant productivity, declining agricultural growth rate, global climate change and the growing anxiety over food security. The ever increasing human population adds to the threat of global food security under the present grim condition of changing climate, global warming, increased pollution, biotic and abiotic stress, (Maiti, 2011). In order to cope up with these challenges, strengthening the public agricultural research system of India has been increasingly felt in recent years by the research managers, administrators and policy makers of the country. It is well understood that the overall productivity of the agricultural research system largely depends upon the research productivity of individual scientists working under it. Therefore, it is crucial to gauge the present status of research productivity of the scientists and develop suitable policies to enhance it. A number of techniques like h-index (Hirsch, 2005), g-index (Egghe, 2006), AR-index (Jin, 2007), RP-index and CP index (Altmann et al., 2009) have been developed so far as an absolute measure of research productivity. These measures are dependent mainly upon publications, citations and other bibliometric data which sometimes become quite difficult to obtain. As an alternative, some crude measures of research productivity in form of analysing the factors which determine research productivity have also been suggested by some other researchers (Babu and Singh, 1998; Buchmueller et al., 1999). Some researchers have viewed organizational productivity and its effectiveness as a function of team work and collaboration. Bennis (1969) pointed out that team collaboration, participation in decision making and satisfaction with co-workers are essential characteristics for effectiveness of an organization in formalized job setting. Beaver and Rosen (1979) opined that despite some variations among disciplines, working with others for scientific research has become the norm. As evident from these studies, research productivity is a function of both organizational as well as personal factors. The present study aimed at devising a much simpler method of predicting research productivity objectively through some personal factors in general and individual work styles in particular. The basic idea behind undertaking the present research was that it becomes rarely possible for the individual scientists to intervene into the productivity influencing organizational factors as these largely depend 
upon organizational policies, hence associated procedural complexities. On the other hand the personal factors are easier to be self intervened as the personal work styles can be readily appraised, easily monitored and necessarily moulded as required for sustaining a research team. The work style of scientists refers to one's preference for work related approaches to creative problem solving, interpersonal relationship, controlling work, maintaining performance standard, managing time etc. Although there have been studies on management ability (Satya Gopal and Reddy, 2000), managerial styles (Sandhya, 1985) and leadership styles (Singh et al., 2008) of agricultural scientists, no study has been reported so far in India in relation to work styles of scientists. With this view, the present study had been undertaken to design a work style compass to investigate into the different work styles of agricultural scientists and to develop a suitable model to predict research productivity of scientists through the work styles.

\section{Materials and Methods}

The study, conducted during 2008-09 to 2011-12 utilized an expost facto research design. The locale of the study purposively was two differently performing agricultural institutes of India, Indian Agricultural Research Institute (IARI), New Delhi, chosen among the high performing institutes (HPIs) and Chandra Shekhar Azad University of Agriculture and Technology (CSAUA\&T), Kanpur, chosen among the low performing institutes (LPIs). The selection was made following the results of a previous study (Education Times, 2009) and validated by the results of another study (Gupta, 2011) to rank Indian agricultural universities on different parameters. A multistage disproportionate stratified random sampling technique was applied to select a sample of two hundred agricultural scientists $(\mathrm{n}=200)$, hundred each from different cadres of the HPI and LPI.

Work style was operationalized as a measure of one's preference for and pattern of accomplishing tasks indicated through creativity, supportiveness, control over task, quality orientation, and time use efficiency. Except for creativity which was measured using the creativity test (Nagasri, 2000) with necessary modifications, separate Likert type scales were developed to quantify the remaining four variables. The instruments were pilot tested with thirty non-sample respondents and the coefficients of reliability obtained were $0.84,0.82,0.88$, and 0.86 respectively for supportiveness, control over task, quality orientation, and time use efficiency. Content validity of the instruments was established through a panel of twenty five experts comprising of agricultural scientists, research managers and administrators. A research productivity index was developed to find out the research productivity scores of the respondents under the study. Data were collected through in-depth personal interview method. Frequency, percentage, F-test, Pearson's product moment correlation and stepwise multiple regression analysis were undertaken for meaningful interpretation of the collected data.

\section{Results and Discussion}

\subsection{The work style compass}

The work style compass, designed based upon the findings of the study comprised of five dimensions, the two sides of each dimension representing the two extremes of a particular work style (Figure 1). Thus, the respondents scoring low in the given creativity test were supposed to be following routine pattern of work accomplishment, hence their work style has been termed as 'conventional.' Almost half (49.5\%) of the total number of respondents were found to have such work style. It is noteworthy that only $13 \%$ of the respondents followed 'creative' work style (Table 1). Remaining 37.5\% of the respondents were neither very creative nor exactly routine work style followers. The group of scientists showing below average level of creativity perhaps were obsessed with routine tasks that hindered their divergent thinking abilities. In addition to some personality factors, cognitive factors, intrinsic motivation, freedom and knowledge (Woodman et al., 1993 and Amabile, 1997) there may be number of organizational factors to influence creativity of the scientists. As pointed out by Andriopoulos (2001), organizational creativity is affected by five factors, namely, organizational climate, leadership style, organizational culture, resources and skills, and structure and systems of the organization.

As far as supportiveness dimension of work style is concerned, it was found that a majority $(60 \%)$ of the respondents fall in

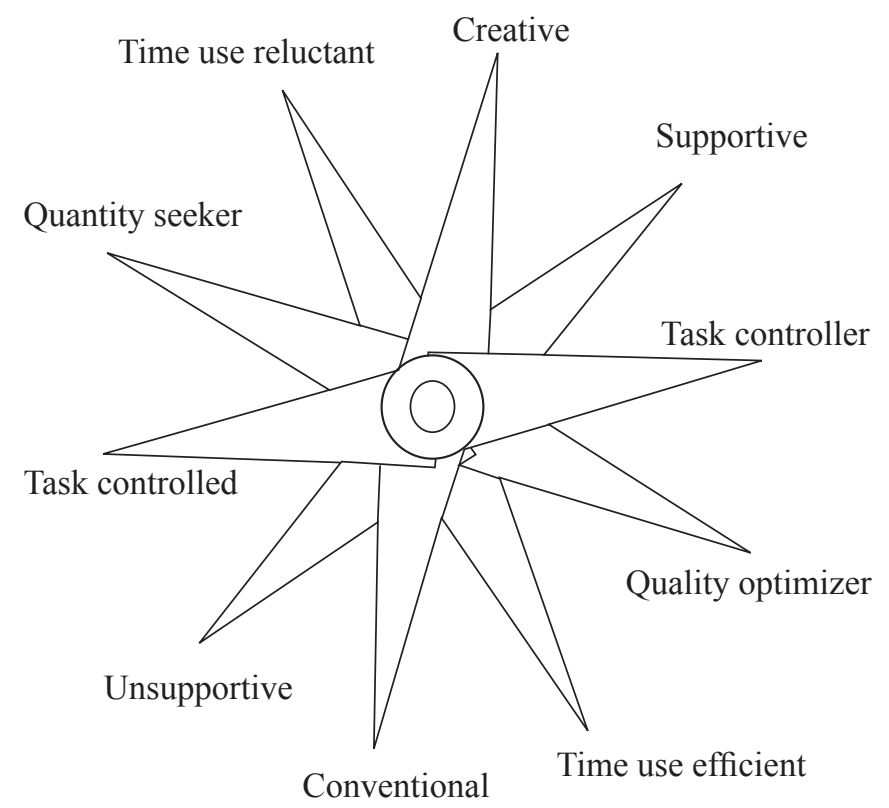

Figure 1: The Work style compass 
the medium category of the supportiveness continuum. A little above one fifth $(22.5 \%)$ and a little below one fifth $(17.5 \%)$ of them fall respectively in the low and high categories (Table 2). Scientists failing to follow the 'supportive' work style were most of the time unable to render job related supports to their fellow scientists. The scientists of the LPI had laid primary thrust on teaching which is an individual activity and does not necessarily require team work. Lack of adequate involvement in multidisciplinary research might have been one of the major reasons for lower level of supportiveness among the group of scientists following the 'unsupportive' work style. It was found that the highest proportion of 'supportive' scientists belonged to the cadre of Principal Scientists from the HPI. By and large, research managerial positions in the HPI were invariably occupied by the Principal Scientists. Serving such positions required highly supportive work style. It may also be a reason for higher level of supportiveness as exhibited by the larger portion of Principal Scientists of the HPI.

The distinguished parity in control over task among the scientists has been illustrated in the work style compass as task controller-task controlled extremes. More than half (57.5\%) of the agricultural scientists under study had a medium level of control over task. More than one fourth $(29 \%)$ of them had considerably low control and remaining $13.5 \%$ of the respondents were found to have higher control over their works (Table 3). Work style of scientists characterized by lower level of control over their task has been named as 'task controlled.' Such work style reflects lack of job autonomy and lack of pro-activeness. Lack of proper planning in advance, lack of regular monitoring at personal level, work interruption and frequent activity switches might be the other reasons for lower control over task.

Table 1: Distribution of respondents according to their level of creativity

\begin{tabular}{lcc}
\hline Category* & \multicolumn{2}{c}{ Distribution of respondents $(\mathrm{n}=200)$} \\
\cline { 2 - 3 } & $\mathrm{f}$ & $\%$ \\
\hline Low (0-7.35) & 99 & 49.5 \\
Medium (7.35-11.13) & 75 & 37.5 \\
High ( $\geq 11.13)$ & 26 & 13.0 \\
\hline "stratified following cumulative cube root frequency method
\end{tabular}

Table 2: Distribution of respondents according to their level of supportiveness

\begin{tabular}{lcc}
\hline Category & \multicolumn{2}{c}{ Distribution of respondents $(\mathrm{n}=200)$} \\
\cline { 2 - 3 } & $\mathrm{f}$ & $\%$ \\
\hline Low (0-10.86) & 45 & 22.5 \\
Medium (10.86-14.16) & 120 & 60.0 \\
High (14.16-32.00) & 35 & 17.5 \\
\hline "stratified following cumulative cube root frequency method
\end{tabular}

With respect to quality orientation, it was observed that $33 \%$ of the agricultural scientists had considerably lower level of quality orientation (Table 4). Their work style by and large was quantity oriented. Work style of a large portion of agricultural scientists from the LPI was found to of this type. Such work style followers were termed as 'quantity seekers.' Another category of work style followers were 'quality optimizers' whose inclination towards enhancing quality of their work was very high. Only $22 \%$ of respondents followed such work style. Remaining $45 \%$ of them belonged to the medium category of the quality orientation continuum. The research infrastructure and other research facilities, prevailing performance appraisal system and promotional policy might not have succeeded enough to foster a motivational climate congenial for high quality research work. Quality optimizing work style was mainly prevalent among the scientists from the HPI. High prestige of the institute and a competitive atmosphere of the HPI might have contributed to adoption of a quality oriented work style by the scientists working in that institute.

The study further reveals that work style of about one third $(29.5 \%)$ of the respondents was characterized by time use reluctance leading to lower level of time use efficiency of such scientists. Lack of adequate concern for maintaining deadline and procrastinating behaviour might be among the various reasons for such inefficiency. Lay and Schouwenburg (1993) found that people with higher trait of procrastination were low in setting goal and priority and showed greater likelihood to fail the schedule of personal projects. Majority (55\%) of the respondents however had a medium level of time use efficiency whereas only $15.5 \%$ of them were highly 'time use efficient' (Table 5).

Table 3: Distribution of respondents according to their level of control over task

\begin{tabular}{lcc}
\hline Category & \multicolumn{2}{c}{ Distribution of respondents $(\mathrm{n}=200)$} \\
\cline { 2 - 3 } & $\mathrm{f}$ & $\%$ \\
\hline Low (0-9.14) & 58 & 29 \\
Medium (9.14-12.14) & 115 & 57.5 \\
High (12.14-32.00) & 27 & 13.5 \\
\hline "stratified following cumulative cube root frequency method
\end{tabular}

Table 4: Distribution of respondents according to their level of quality orientation

\begin{tabular}{lcc}
\hline \multirow{2}{*}{ Category } & \multicolumn{2}{c}{ Distribution of respondents $(\mathrm{n}=200)$} \\
\cline { 2 - 3 } & $\mathrm{f}$ & $\%$ \\
\hline Low (0-33.15) & 66 & 33 \\
Medium (33.15-41.05) & 90 & 45 \\
High (41.05-60.00) & 44 & 22 \\
\hline "stratified following cumulative cube root frequency method
\end{tabular}


3.2. Variation in work styles among the cadres of agricultural scientists

The results of a one way analysis of variance confirmed that different cadres of agricultural scientists under study significantly varied in terms of all the work style variablescreativity $[\mathrm{F}(3,196)=20.125, p<0.001]$, supportiveness [F $(3,196)=34.97, p<0.001]$, control over task $[\mathrm{F}(3,196)=7.56$, $p<0.001]$, quality orientation $[\mathrm{F}(3,196)=32.5, p<0.001]$ and time use efficiency $[\mathrm{F}(3,196)=18.15, p<0.001]$ (Table 6).

\subsection{Degree of association between research productivity and work style variables}

In order to predict research productivity though work style, it was important to gauge the degree of association between research productivity and the work style variables. A correlation analysis depicts that research productivity of agricultural scientists had positive and statistically significant correlation with all the work style variables under study-creativity (0.75), supportiveness ( 0.65$)$, control of work ( 0.7$)$, quality orientation (0.81), and time use efficiency (0.82) as indicated by the significant correlation coefficients (r) (Table 7). The data in Table 7 further indicates the extent of association between research productivity and the work style variables as depicted by the results of a stepwise multiple regression analysis. It could be observed that the Durbin-Watson statistic (1.74) was close to 2 . Hence, the assumption of tenability of the independent errors had been satisfactorily met. The regression coefficients (b) associated with creativity (0.20), supportiveness (0.04), control of work (0.13), quality orientation (0.08) and time use efficiency $(0.08)$ were all found positive indicating a positive relationship of these predictor variables with the dependent variable research productivity.

Creativity $(\mathrm{t}=8.319, p<0.001)$, control of work $(\mathrm{t}=3.796$,

Table 5: Distribution of respondents according to their time use efficiency

\begin{tabular}{lcc}
\hline Category & \multicolumn{2}{c}{ Distribution of respondents $(\mathrm{n}=200)$} \\
\cline { 2 - 3 } & $\mathrm{f}$ & $\%$ \\
\hline Low (0-26.65) & 59 & 29.5 \\
Medium (26.65-35.12) & 110 & 55 \\
High (35.12-52.00) & 31 & 15.5 \\
\hline
\end{tabular}

"stratified following cumulative cube root frequency method $p<0.001)$ quality orientation $(\mathrm{t}=6.493, p<0.001)$, and time use efficiency ( $\mathrm{t}=5.677, p<0.001$ ), were found to be significant predictors of research productivity but supportiveness $(\mathrm{t}=1.333$, $p>0.05$ ) was found to make insignificant contribution towards predicting research productivity. As evident from the magnitude of $\beta$ as well as $t$ values, creativity had the highest impact in terms of contribution in research productivity followed by quality orientation, time use efficiency, and control of work. Thus, the regression equation finally derived for predicting research productivity based upon the work style variables could be represented as: $Y=0.292 \mathrm{X}_{1}+0.316 \mathrm{X}_{2}+0.306 \mathrm{X}_{3}+0.155$ $\mathrm{X}_{4}-5.956+0.386$, (where, $\mathrm{Y}$ stands for research productivity; $\mathrm{X}_{1}$, $\mathrm{X}_{2}, \mathrm{X}_{3}, \mathrm{X}_{4}$ respectively stand for time use efficiency, creativity, quality orientation, and control of work; constant $=-5.956$, and standard error $=0.386$ ).

The variable supportiveness was not included in the regression equation because of the statistically insignificant contribution produced by the variable in predicting research productivity. The value of adjusted $\mathrm{R}^{2}$ (coefficient of determination) was found to be as high as 0.83 implying that the four variables- creativity, quality orientation, time use efficiency and control of work accounted for about $83 \%$ variability in research productivity. The significant $\mathrm{F}$ ratio $[\mathrm{F}(4,195)=247.664, p<0.001]$ as found in the last step of stepwise multiple regression analysis indicated that the regression model had significantly predicted the outcome variable research productivity.

3.4. Predicting research productivity through the work style compass

Table 7: Correlation and multiple regression coefficients of research productivity with work style variables $(n=200)$

\begin{tabular}{lcccc}
\hline Variable & $\begin{array}{c}\text { Correla- } \\
\text { tion coef- } \\
\text { ficient }(\mathrm{r})\end{array}$ & $\begin{array}{c}\text { Regres- } \\
\text { sion coef- } \\
\text { ficient }(\mathrm{b})\end{array}$ & $\begin{array}{c}\text { Standard- } \\
\text { ized coef- } \\
\text { ficient }(\beta)\end{array}$ & $\mathrm{t}$ \\
\hline Creativity & $0.75^{* *}$ & 0.20 & 0.32 & $8.32^{* *}$ \\
Supportiveness & $0.65^{* *}$ & 0.04 & 0.06 & 1.33 \\
Control of work & $0.70^{* *}$ & 0.13 & 0.16 & $3.80^{* *}$ \\
Quality orientation & $0.81^{* *}$ & 0.08 & 0.31 & $6.49^{* * *}$ \\
Time use efficiency & $0.82^{* *}$ & 0.08 & 0.29 & $5.68^{* *}$ \\
\hline${ }^{* *}$ significant at $\left.p=.01\right) ; \mathrm{F}=247.66^{* *}$ with $(4,195) \mathrm{df}$; Adjusted \\
$\mathrm{R}^{2}=0.83$; Standard error of the estimate=0.69; Durbin- \\
Watson=1.74
\end{tabular}

Table 6: Calculated $\mathrm{F}$ values depicting significance of variation in work style among the cadres of respondents under study $(\mathrm{n}=200)$

\begin{tabular}{lccc}
\hline Test group & Work style variable & $\mathrm{F}$ & $\mathrm{p}$ \\
\hline Associate Professor (LPI) & Creativity & 20.13 with $(3,196) \mathrm{df}$ & $<0.001$ \\
Professor (LPI) & Supportiveness & 34.97 with $(3,196) \mathrm{df}$ & $<0.001$ \\
Senior Scientist (HPI) & Control over task & 7.56 with $(3,196) \mathrm{df}$ & $<0.001$ \\
Principal Scientist (HPI) & Quality orientation & 32.5 with $(3,196) \mathrm{df}$ & $<0.001$ \\
& Time use efficiency & 18.15 with $(3,196) \mathrm{df}$ & $<0.001$ \\
\hline
\end{tabular}


As envisaged by the findings of the study, a strong positive correlation between research productivity of agricultural scientists and different work style variables existed. It implies that research productivity of scientists varied according to their adopted work styles. Scientists following routine work styles had lower level of research productivity when compared to the creative work style followers. Scientists with creative work style were intrinsically motivated. They looked for originality to formulate research projects and applied novel approaches to solve research problems. They tried to diversify activities with new ideas and thoughts. Creative scientists are usually hard working, obsessed with their work and have intense devotion to that (Roe, 1953). Conventional work style followers perhaps were obsessed with repetitive activities and restricted their efforts within limited sphere of tasks. As a result, they were unable to obtain a higher level of research productivity. Agricultural scientists who were supportive to their fellow scientists and subordinates were more productive than those with unsupportive kind of work styles. In contrary to unsupportive work style followers who worked in isolation most of the time, supportive scientists wanted to be productive research team members and as a result they were able to reap the benefits of team work and collaboration in form of coauthorship of publications, co-ownership of research projects and co-coordination of training and various other extension activities that might have added to their total productivity scores. Significant difference in productivity could also be observed between the scientists with higher and lesser control over task as indicated by the findings of the study. Scientists seeking job autonomy, planning their day to day project activities in advance and monitoring the work at personal level had higher control over their tasks. They always strived for maintaining superior performance standard. On the other hand lower control over task was supposed to be accompanied by impoverished planning, task overload and increased job strain resulting in poor performance and productivity.

The results of the correlation and regression analysis further reveals that scientists with higher quality orientation were much more productive than the scientists with lower quality orientation. Quality orientation of scientists might have led to accomplishment of high quality research work and communication of the findings in national and international research journals with higher rating and impact factors that might have helped them in securing higher publication scores. Due to high quality of publication, they might have received higher number of citations and thereby higher professional recognition. Besides, scientists with higher quality orientation might have received more number of awards for their high quality research works. All these added to their overall research productivity scores.
Productivity scores may have increased with higher time use efficiency of agricultural scientists as indicated by the significant correlation coefficient between research productivity and time use efficiency. Agricultural scientists with lower time use efficiency scores may be considered as time use reluctant. Followers of such work style might be indifferent to prioritize activities, prone to procrastination, little concerned of maintaining deadline and rarely using daily work schedule. Time utilization pattern to have positive relationship with research efficiency was reported by Tripathi and Varsha (2007).

\section{Conclusion}

It brings into focus a work style compass to elucidate the work styles prevailing among the agricultural scientists of India. The revelation of significant positive relationship between research productivity and work style variables entails the need for certain work style interventions to be made for optimizing individual research productivity and thereby organizational research effectiveness as a whole. Conducting 'work style sensitization workshop' at regular intervals at the institute level along with Organizational Development (OD) interventions are crucial for enhancing research productivity of agricultural scientists.

\section{References}

Altmann, J., Abbasi, A., Hwang, J., 2009. Evaluating the productivity of researchers and their communities: The RP-Index and the CP-Index. International Journal of Computer Science and Applications 6(2), 104-118.

Amabile, T.M., 1997. Motivating creativity in organizations: on doing what you love and loving what you do. California Management Review 40, 39-58.

Andriopoulos, C., 2001. Determinants of organizational creativity: a literature review. Management Decision 39(10), 834-840.

Anon, 2009. Ranking of Indian universities: An Education Times-Gfk-mode study. Education Times, The Times of India. Weekly, March 30, 2009, 2.

Babu, A.R., Singh, Y.P., 1998. Determinants of research productivity. Scientometrics 43(3), 309-329.

Beaver, D., Rosen, R., 1979. Studies in scientific collaboration: part II-scientific co authorship, research productivity and visibility in the French scientific elite. Scientometrics 1, 133-149.

Bennis, W.G., 1969. Organization Development: Its Nature, Origins, and Prospects. Reading, Massachusetts. Addition-Wesley publishing company.

Buchmueller, T., Dominitz, J., Hansen, L., 1999. Graduate training and the early career productivity of Ph.D. 
Economists. Economics of Education Review 14, 65-77.

Egghe, L., 2006. Theory and practice of the g-index. Scientometrics 69(1), 131-152.

Gupta, B.M., 2011. Ranking of Indian institutions in agriculture and allied sciences for their research output during 1999-2008. Annals of Library and Information Studies $58,63-70$.

Hirsch, J.E., 2005. An index to quantify an individual's scientific research output. Proceedings of the National Academy of Sciences 102(46), 16569 -16572.

Jin, B.H., 2007. The AR-index: complementing the h-index. ISSI Newsletter 3(1), 6.

Lay, C.H., Schouwenburg, H.C., 1993. Trait procrastination, time management, and academic behaviour. Journal of Social Behavior and Personality 8, 647-662.

Maiti, R., 2011. Technology development for conservation, propagation and adaptation of native crops to biotic and abiotic stress factors: concept, hypothesis and confirmation. International Journal of Bioresource and Stress Management 2(2), I-IV.

Nagasri, K., 2000. Assessment of creative potential and practices of extension personnel. Ph. D. thesis, Unpublished. New
Delhi: Indian Agricultural Research Institute.

Roe, A., 1953. A psychological study of eminent psychologists and anthropologists, and a comparison with biological and physical scientists. Psychological Monograph 67(2), 1-55.

Sandhya, P.N., 1985. Managerial styles and research productivity. Ph. D. thesis, Unpublished. New Delhi: Indian Agricultural Research Institute.

Satya Gopal, P.V., Reddy, B.L., 2000. Rating scale to measure management abilities of scientists heading research stations. Indian Journal of Extension Education 36(3\&4), 167-168.

Singh, N., Sinha, B.P., Vijayaragavan, K., 2008. Motivational training programme: Effects on the development of leadership styles in agricultural scientists. Indian Research Journal of Extension Education 8(1), 25-27.

Tripathi, B.D., Varsha, 2007. Factors contributing to efficiency of personnel working in a research organization-a case study. Journal of Agricultural Extension Management $8(2), 85-96$.

Woodman, R.W., Sawyer, J.E., Griffin, R.W., 1993. Toward a theory of organizational creativity. Academy of Management Review 18(2), 293-321. 